NISSUNA UMANA INVESTIGAZIONE SI PUO DIMANDARE VERA SCIENZIA S'ESSA NON PASSA PER LE MATEMATICHE DIMOSTRAZIONI LEONARDO DA VINCI

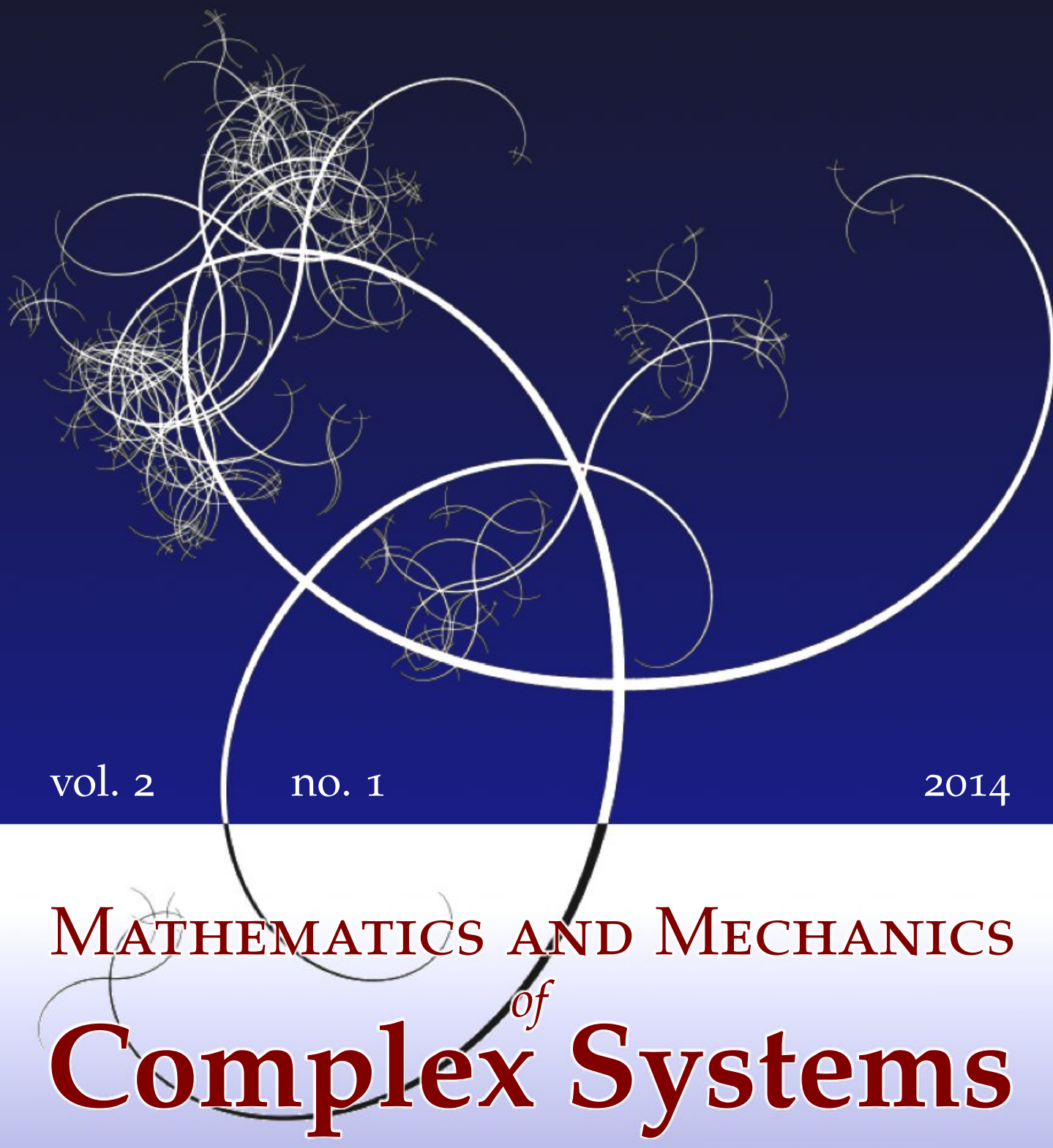

Andrea Colagrossi, Giorgio Graziani and Mario Pulvirenti

PARTICLES FOR FLUIDS:

SPH VERSUS VORTEX METHODS 


\title{
PARTICLES FOR FLUIDS: SPH VERSUS VORTEX METHODS
}

\author{
Andrea Colagrossi, Giorgio Graziani And Mario Pulvirenti
}

We review the basic ideas underlying two popular particle methods for fluids: smoothed-particle hydrodynamics and the vortex method. We discuss convergence problems, numerical schemes, and examples of practical simulations.

\section{Introduction}

The Lagrangian form of the Navier-Stokes equations can be approximated with particle methods by replacing derivative operators through integral operators which are discretized on the particle locations. These particles carry the physical properties of a fluid dynamic system. Their trajectories, as well as the evolution of the transported quantities, are evaluated through the solution of ordinary differential equations. Particle methods such as vortex methods (VMs) and smoothed-particle hydrodynamics (SPH) present interesting features which allow the simulation of engineering problems involving complex interface dynamics such as vortex sheets and free-surface deformations (for a more general discussion of particle methods see, for example, [Koumoutsakos 2005]). SPH is a particle method introduced by Lucy [1977] and further developed by Monaghan (see, for example, [Monaghan 1992]) to study self-gravitating fluids. The basic idea of the method is to consider the fluid as an ensemble of (smooth) particles whose evolution simulates that of the fluid under consideration. Each particle has a form factor (a symmetric, regular, nonnegative function centered on the particle position) which simulates the mass distribution around the center of each particle. Moreover the particles move in the force field generated by the whole particle system.

For many years SPH was used for astronomical problems; later, it was applied to several physics and engineering problems, ranging from solid mechanics to multiphase flows. Monaghan [1994] first applied it for simulating free-surface inviscid flows. Compared to the Eulerian finite difference method, SPH can handle breaking and fragmentation phenomena in a more natural way, due to its Lagrangian nature.

MSC2010: primary 35Q30, 35Q35, 76D17, 35Q31; secondary 35L60, 35Q70.

Keywords: particle methods, smoothed-particle hydrodynamics, vortex method, convergence, viscous flows. 
Thanks to this valuable feature, interest in SPH has grown quickly in the last decade and several researchers have devoted their efforts to improving this method.

Clearly the basic requirement for this particle method is its convergence to the right solution of the fluid dynamic equations when the number of particles diverges. In this paper we want to discuss this issue in connection with Vlasov dynamics, which is strictly related to problem at hand.

Another well-known method discussed in the present paper is the vortex method (VM), which works for two-dimensional incompressible flows. This is also a particle approximation of the incompressible Euler and Navier-Stokes equations in the vorticity formalism. It is conceptually related to the SPH method because both approximate the fluid equations in a suitable mean-field limit. Here we want to discuss these methods exploiting this aspect.

As we shall discuss in some detail in the sequel, SPH and the VM seem to be effective in approaching practical problems. In this work numerical solutions of viscous flows obtained with both methods are discussed showing the benefits and drawbacks of the two solvers. In particular, using the same spatial resolution, the VM provides numerical solutions with higher accuracy and lower CPU costs than does SPH. However, it is important to underline that the latter method can be applied much more easily on a wider range of engineering problems (free-surface, multiphase, interfaces, compressible flows, etc.), while for the VM even extension from two to three-dimensional framework is not straightforward. Therefore, the choice between the two particle methods is still strongly problem dependent. Another important point regards the parallelization of the different algorithms. Since the SPH model is based on short-range interactions, it is quite easy to parallelize in a very efficient way (see, for example, [Marrone et al. 2012]). This is not the case for the VM, where the evaluation of velocity fields requires more effort to parallelize (see, for example, [Barnes and Hut 1986; Antonuccio-Delogu et al. 1996]).

The plan of the paper is as follows. In Section 2 we introduce SPH and the VM as regards their general structure and the convergence problems. In Section 3 we discuss some numerical schemes suited for practical implementations of the two methods. Finally, Section 4 is devoted to examples of practical simulations.

\section{Convergence of particle methods}

2.1. Euler equation. In the present subsection we introduce the basic equations we want to investigate. Let us start with the isentropic Euler equation for a pressure law $p=(1 /(\alpha+2)) \rho^{\alpha+2}$, where $p$ is the pressure and $\rho$ is the density:

$$
\begin{cases}\frac{\partial \rho}{\partial t}+\nabla \cdot(\rho u)=0, & \frac{\partial u}{\partial t}+(u \cdot \nabla u)+\rho^{\alpha} \nabla \rho=0, \\ \rho(x, 0)=\rho_{0}(x), & u(x, 0)=u_{0}(x) .\end{cases}
$$


Here $u=u(x, t)$ denotes the velocity field, $x \in \mathbb{R}^{d}, d=2,3$, the dimension of the physical space, and $t \in \mathbb{R}$ the time.

The incompressible Euler equation reads

$$
\frac{\partial u}{\partial t}+(u \cdot \nabla u)+\rho_{0}^{-1} \nabla p=0, \quad \nabla \cdot u=0, \quad u(x, 0)=u_{0}(x) .
$$

Here we are considering the fluid invading the whole space. However most of the applications we are interested in the fluids are confined in domains with boundaries, in which also viscous effects are not negligible. We discuss these important aspects later on.

2.2. The Vlasovequation. When dealing with large weakly interacting particle systems in stellar dynamics or in the physics of plasmas, we often use the following evolution equation for $f(x, v, t)$, the fraction of particles in the cell of the phase space around $(x, v)$ of size $d x d v$ (or the probability density of finding a given particle in $(x, v))$ :

$$
\left(\partial_{t}+v \cdot \nabla_{x}+F \cdot \nabla_{v}\right) f(x, v, t)=0 .
$$

Here $x \in \mathbb{R}^{d}$ denotes the position and $v \in \mathbb{R}^{d}$ the velocity of a test particle, while $t \in \mathbb{R}$ denotes the time, with $d=2,3$ the dimension of the physical space.

Note that (2-3) is the usual Liouville equation for a single particle in a force field $F=F(x, t)$, so that (2-3) is solved by the formula

$$
f(x, v, t)=f_{0}\left(\Psi^{-t}(x, v)\right),
$$

or, equivalently,

$$
\int d x d v f(x, v, t) g(x, v)=\int d x d v f_{0}(x, v) g\left(\Psi^{t}(x, v)\right),
$$

where $f_{0}$ is the initial datum for $(2-3), \Psi^{t}(x, v)=(x(t), v(t))$ is the flow solution to the ordinary differential system

$$
\dot{x}(t)=v(t), \quad \dot{v}(t)=F(x(t), t),
$$

with initial datum $\Psi^{0}(x, v)=(x, v)$, and $g$ is any bounded continuous function.

Equation (2-3) differs from the Liouville equation because $F$ is not known a priori, but depends on the solution itself, via the self-consistent formula

$$
F(x, t)=\int d x K(x-y) \rho(y, t) d y,
$$

where

$$
\rho(x, t)=\int d v f(x, v, t)
$$

is the spatial density and $K: \mathbb{R}^{d} \rightarrow \mathbb{R}^{d}$ is a given kernel. In other words (2-3) is a 
nonlinear equation because the vector field $F$ depends on the solution $f$.

Clearly the interesting case is when the force kernel $K$ arises from a potential, namely

$$
K(x)=-\nabla \varphi(x) .
$$

If $\varphi$ is assumed to be smooth, it is possible to show that there exists a unique solution to the initial value problem associated with (2-3).

Following [Dobrušin 1979] (see also [Braun and Hepp 1977; Neunzert 1984]), we can establish the following result:

Theorem 1. Consider the N-particle evolution

$$
\left\{\begin{array}{l}
\dot{x}_{i}(t)=v_{i}(t), \\
\dot{v}_{i}(t)=-\frac{1}{N} \sum_{\substack{1 \leq j \leq N \\
j \neq i}} \nabla \varphi\left(x_{i}(t)-x_{j}(t)\right), \\
x_{i}(0)=x_{i}, \quad v_{i}(0)=v_{i},
\end{array}\right.
$$

where $i=1, \ldots, N$. Introduce an empirical measure defined on the one-particle phase space by

Then if

$$
\mu_{N}(d x, d v ; t)=\frac{1}{N} \sum_{i=1}^{N} \delta\left(x-x_{i}(t)\right) \delta\left(v-v_{i}(t)\right) d x d v
$$

$$
\lim _{N \rightarrow \infty} \int d \mu_{N}(x, v ; 0) g(x, v)=\int f_{0}(x, v) g(x, v) d x d v,
$$

for all functions $g$ continuous and bounded, then, for all $t>0$,

$$
\lim _{N \rightarrow \infty} \int d \mu_{N}(x, v ; t) g(x, v)=\int f(x, v, t) g(x, v) d x d v,
$$

where $f(t)$ solves (2-3) with initial datum $f_{0}$.

We do not present the proof of this theorem, but some comments are in order. Let $M$ be the space of the Borel probability measure on $\mathbb{R}^{d}$. We first observe that there exists a unique measure-valued solution $t \rightarrow v(t) \in \mathcal{M}$ to (2-3), in the following weak form (which does not require any smoothness of $v$ ), for any smooth test function $g$ :

$$
\frac{d}{d t}\langle v, g\rangle=\left\langle\nu, v \cdot \nabla_{x} g\right\rangle+\left\langle v, F \cdot \nabla_{v} g\right\rangle,
$$

where

$$
\langle v, g\rangle=\int d x \int d v g(x, v) v(d x, d v),
$$

and $F=K * v$ is the self-consistent force.

Using integration by parts, it is easy to show that (2-13) holds whenever $f(t)$ is a classical solution to (2-3) and $v(d x, d v, t)=f(t) d x d v$. 
Moreover a trivial computation also shows that $\mu_{N}$ is a weak solution to (2-13). Therefore Theorem 1 says precisely that the solution to the Vlasov equation at any fixed time $t>0$, is continuous with respect to the initial data, for the topology induced by the weak convergence of the measures, expressed by condition (2-11).

There is a fruitful connection between Vlasov dynamics and the solutions of the problems given by (2-1) and (2-2), which we shall discuss in the next sections.

2.3. The SPH model. Let us start to consider the Euler problem (2-1) for the particular case $\alpha=0$ :

$$
\left\{\begin{array}{l}
\rho_{t}+\nabla \cdot(\rho u)=0, \\
u_{t}+(u \cdot \nabla u)+\nabla \rho=0, \\
\rho(x, 0)=\rho_{0}(x), \quad u(x, 0)=u_{0}(x) .
\end{array}\right.
$$

In Lagrangian form it reads

$$
\left\{\begin{array}{l}
\ddot{\Phi}_{t}(x)=-\nabla \rho\left(\Phi_{t}(x), t\right), \\
\int \rho(x, t) g(x)=\int \rho_{0}(x) g\left(\Phi_{t}(x)\right), \\
\Phi_{0}(x)=x, \quad \dot{\Phi}_{0}(x)=u_{0}(x),
\end{array}\right.
$$

where $g$ is a bounded continuous function and $\Phi_{t}: \mathbb{R}^{d} \times \mathbb{R}^{+} \rightarrow \mathbb{R}^{d}$ is the solution flow.

The Eulerian velocity is recovered via the formula

$$
u\left(\Phi_{t}(x), t\right)=\dot{\Phi}_{t}(x)
$$

Recall that a solution to the Lagrangian problem (2-15) (also in Eulerian form, (2-14)) exists only locally in time; see, for example, [Majda 1984].

Next we regularize the problem by setting

$$
\left\{\begin{array}{l}
\ddot{\Phi}_{t}(x)=-\nabla\left(\delta_{\varepsilon} * \rho\right)\left(\Phi_{t}(x), t\right), \\
\int \rho(x, t) g(x)=\int \rho_{0}(x) g\left(\Phi_{t}(x)\right), \\
\Phi_{0}(x)=x, \quad \dot{\Phi}_{0}(x)=u_{0}(x),
\end{array}\right.
$$

where the form factor $\delta_{\varepsilon}$ is a positive, smooth approximation of the $\delta$ function. Here $\varepsilon$ is a small positive parameter such that

$$
\delta_{\varepsilon} \rightarrow \delta
$$

in the sense of the weak convergence of the measures (2-11), as $\varepsilon \rightarrow 0$.

We note that, as we shall see in a moment, problem (2-16) has a global solution (even for initial densities $\rho_{0}$ which are measures). 
The connection with the Vlasov equation discussed in the last section is clear. Indeed consider the solution flow (2-6) to the Vlasov equation with

$$
F=K * \rho, \quad K=-\nabla \delta_{\varepsilon},
$$

and with initial datum

$$
f_{0}(x, v) d x d v=\rho_{0}(x) \delta\left(v-u_{0}(x)\right) d x d v .
$$

Then, setting

$$
\left(\Phi_{t}(x), \dot{\Phi}_{t}(x)\right)=\Psi^{t}\left(x, u_{0}(x)\right)
$$

the time evolved measure $f(x, v, t) d x d v$ satisfies

$$
\begin{aligned}
\int f(x, v, t) g(x, v) d x d v & =\int f_{0}(x, v) g\left(\Psi^{t}(x, v)\right) d x d v \\
& =\int d x d v \rho_{0}(x) \delta(v-u(x)) g\left(\Psi^{t}(x, v)\right) \\
& =\int d x d v \rho_{0}(x) g\left(\Phi_{t}(x), \dot{\Phi}_{t}(x)\right),
\end{aligned}
$$

which implies (2-16). In fact, if $g$ depends only on $x$, we get the second formula of (2-16), while the first one follows by the obvious remark that $\ddot{\Psi}_{t}(x, u(x))=\ddot{\Phi}_{t}(x)$. In other words, the solution to the problem (2-16) is a particular solution to the Vlasov equation for the special initial datum (2-17). Note that although such a solution, due to the smoothness of $\delta_{\varepsilon}$, does exist for any arbitrary time $t$, it not necessarily take the form

$$
f(x, v) d x d v=\rho(x, t) \delta(v-u(x, t)),
$$

where the velocity and the density fields $u(x, t)$ and $\rho(x, t)$ satisfies the equivalent of (2-14) with the corresponding regularized pressure field. Actually, by using the well-known equivalence between the Lagrangian and the (regularized) Eulerian pictures, this is guaranteed only locally in time, namely in the existence time interval of a smooth solution to the regularized Euler equations.

The SPH model in the present context is the $N$-particle system satisfying

$$
\left\{\begin{array}{l}
\dot{x}_{i}(t)=v_{i}(t), \\
\dot{v}_{i}(t)=-\frac{1}{N} \sum_{\substack{1 \leq j \leq N \\
j \neq i}} \nabla \delta_{\varepsilon}\left(x_{i}(t)-x_{j}(t)\right), \\
x_{i}(0)=x_{i}, \quad v_{i}(0)=u_{0}\left(x_{i}\right),
\end{array}\right.
$$

for $i=1, \ldots, N$, and

$$
\mu_{N}(d x, t)=\sum_{i=1}^{N} \delta\left(x-x_{i}(t)\right) d x .
$$


By Theorem 1, we have

$$
\mu_{N}(d x, t) \rightarrow \rho(x, t)
$$

weakly, as $N \rightarrow \infty$ for a fixed $\varepsilon$, where $\rho$ solves (2-16).

We now analyze the general case including $\alpha \neq 0$. The Lagrangian form is

$$
\left\{\begin{array}{l}
\ddot{\Phi}_{t}(x)=-\rho^{\alpha} \nabla \rho\left(\Phi_{t}(x), t\right), \\
\int \rho(x, t) g(x)=\int \rho_{0}(x) g\left(\Phi_{t}(x)\right), \\
\Phi_{0}(x)=x, \quad \dot{\Phi}_{0}(x)=u_{0}(x),
\end{array}\right.
$$

while its regularized version is

$$
\left\{\begin{array}{l}
\ddot{\Phi}_{t}(x)=-\left(\delta_{\varepsilon} * \rho\right)^{\alpha} \nabla\left(\delta_{\varepsilon} * \rho\right)\left(\Phi_{t}(x), t\right), \\
\int \rho(x, t) g(x)=\int \rho_{0}(x) g\left(\Phi_{t}(x)\right), \\
\Phi_{0}(x)=x, \quad \dot{\Phi}_{0}(x)=u_{0}(x) .
\end{array}\right.
$$

The SPH method can be suitably modified to give

$$
\left\{\begin{array}{l}
\dot{x}_{i}(t)=v_{i}(t), \\
\dot{v}_{i}(t)=-\frac{1}{N} \sum_{\substack{1 \leq j \leq N \\
j \neq i}}\left(\frac{1}{N} \sum_{\substack{1 \leq k \leq N \\
k \neq i}} \delta_{\varepsilon}\left(x_{i}(t)-x_{k}(t)\right)\right)^{\alpha} \nabla \delta_{\varepsilon}\left(x_{i}(t)-x_{j}(t)\right), \\
x_{i}(0)=x_{i}, \quad v_{i}(0)=u_{0}\left(x_{i}\right),
\end{array}\right.
$$

where $i=1, \ldots, N$.

It is possible to adapt the techniques we have discussed so far for the case $\alpha=$ 0 to the present case. All the convergence results we have discussed so far are described in detail in [Mas-Gallic and Raviart 1987; Oelschläger 1991; Di Lisio 1995; Di Lisio et al. 1997; 1998; Ben Moussa and Vila 2000; Ben Moussa 2006].

Actually the SPH models we have introduced approximate problem (2-25) which, in Eulerian form, reads

$$
\left\{\begin{array}{l}
\partial \rho / \partial t+\nabla \cdot(\rho u)=0, \\
\partial u / \partial t+(u \cdot \nabla u)+\left(\rho * \delta_{\varepsilon}\right)^{\alpha} \nabla\left(\rho * \delta_{\varepsilon}\right)=0, \\
\rho(x, 0)=\rho_{0}(x), \quad u(x, 0)=u_{0}(x)
\end{array}\right.
$$

The last step is to show that the solution of (2-27) converges to that of (2-1) when the parameter $\varepsilon$ vanishes. This is It is rather technical, but it can be done; see [Di Lisio et al. 1997] for details.

2.4. The vortex model. Let us consider the Euler equation (2-2) in the plane. Define the vorticity $\omega=\omega(x, t)=\partial_{x_{1}} u_{2}-\partial_{x_{2}} u_{2}$ (which is a scalar quantity in the 
present context). Then we obtain

$$
\left(\partial_{t}+u \cdot \nabla\right) \omega(x, t)=0 .
$$

Here $x=\left(x_{1}, x_{2}\right) \in \mathbb{R}^{2}, t \in \mathbb{R}^{+}$, and $u=u(x, t) \in \mathbb{R}^{2}$ is the velocity field. Due to the incompressibility condition $\nabla \cdot u=0$, we can reconstruct the velocity field $u$ from the vorticity profile $\omega$. The result is

$$
u=\nabla^{\perp} \psi, \quad \psi=-\Delta^{-1} \omega .
$$

Explicitly:

$$
u=K * \omega, \quad K(x)=\nabla^{\perp} g(x)=-\frac{1}{2 \pi} \frac{x^{\perp}}{|x|^{2}},
$$

where

$$
g(x)=-\frac{1}{2 \pi} \log |x|
$$

is the fundamental solution for the Poisson equation in the plane.

There is an evident similarity between (2-28) and the Vlasov equation (2-3) which suggests the following particle model:

$$
\dot{x}_{i}(t)=\frac{1}{N} \sum_{\substack{1 \leq j \leq N \\ j \neq i}} K\left(x_{i}(t)-x_{j}(t)\right), \quad x_{i}(0)=x_{i}, \quad i=1, \ldots, N .
$$

A preliminary difficulty in dealing with (2-31) is the singularity of $K(x)$ for $x$ close to 0 . Regularizing the divergence at the origin via a smooth kernel $K_{\varepsilon}$, we substitute (2-31) by

$$
\dot{x}_{i}(t)=\frac{1}{N} \sum_{\substack{1 \leq j \leq N \\ j \neq i}} K_{\varepsilon}\left(x_{i}(t)-x_{j}(t)\right), \quad x_{i}(0)=x_{i}, \quad i=1, \ldots, N .
$$

Theorem 1 allows us to show that the empirical measure

$$
\mu_{N}(d x ; t)=\sum_{i=1}^{N} \delta\left(x-x_{i}(t)\right) d x
$$

is close to $\omega_{\varepsilon}$ which solves

$$
\left(\partial_{t}+u_{\varepsilon} \cdot \nabla\right) \omega_{\varepsilon}(x, t)=0,
$$

where

$$
u_{\varepsilon}=K_{\varepsilon} * \omega_{\varepsilon} .
$$

As before the last step is to show that $\omega_{\varepsilon} \rightarrow \omega$ where $\omega$ solves (2-28).

In the present context we are considering $\omega$ as a probability measure, however the generalization to arbitrary signed measure is straightforward. 
All these steps can be performed, as discussed in [Marchioro and Pulvirenti 1982; 1984; 1994].

The literature on the vortex model is huge and it would be hopeless to cover it exhaustively. We mention only that the vortex system has been largely investigated from the point of view of the general theory of the Hamiltonian dynamical system, see, for instance, [Newton 2001].

When the fluid is confined in domain with a boundary, say $\Omega$, the VM can be easily extended to describe this situation. It is enough to replace $K$ with $K_{\Omega}$, where

$$
K_{\Omega}(x)=\nabla^{\perp} g_{\Omega}(x),
$$

with $g_{\Omega}$ being the fundamental solution of the Poisson equation in the domain $\Omega$, with the boundary conditions constantly zero.

Note that the usual impermeability condition for the incompressible Euler flow, namely

$$
u(x, t) \cdot n=0 \quad \text { on } \partial \Omega,
$$

where $n$ is the normal to the surface $\partial \Omega$, is automatically satisfied (see, for example, [Marchioro and Pulvirenti 1994]).

Moreover, when the viscous effects are not negligible, we have to consider the Navier-Stokes equation, which, in terms of vorticity, reads

$$
\left(\partial_{t}+u \cdot \nabla\right) \omega(x, t)=v \nabla^{2} \omega \text { for all } x \in \Omega .
$$

Here $v>0$ denotes the kinematic viscosity coefficient.

When $\Omega=\mathbb{R}^{2}$ the viscosity effect can be included in the VMs just by adding $N$ independent Brownian motions to (2-31). This idea goes back to Chorin [1973]. A mathematical analysis of this approach can be found in [Marchioro and Pulvirenti 1982; 1984].

However the vorticity formalism for the Navier-Stokes equation, while convenient as regards the possibility of a particle approximation in terms of stochastic particle systems or in terms of other methods (see the numerical scheme implemented later on), exhibits subtle difficulties in describing the boundary conditions. Indeed the no-slip condition $u=0$ on the boundary is not easily read as a simple condition on the behavior of the vorticity field $\omega$ on the boundary. As we said we have automatically $u(x, t) \cdot n=0$, but we have also to ensure that the tangential component of the velocity must vanish.

Again going back to Chorin [1973], this problem can be overcome by assuming the Neumann boundary condition for the Laplace operator appearing on the righthand side of (2-36). This preserves the total amount of vorticity. Next we can restore the correct boundary conditions by putting a (singular) source of vorticity 
on the boundary, computed in such a way that the tangential component of the velocity on the boundary vanishes.

A rigorous mathematical analysis of this procedure can be found in [Benfatto and Pulvirenti 1984; Boldrighini and Buttà 2011].

From a practical view point a numerical scheme can be implemented by the use of the so-called Chorin-Marsden product formula, which consists in the following procedure. First we evolve the flow by the Euler equation, for a very small time $h$. Then we restore the correct boundary conditions by adding a vortex sheet to compensate for the tangential part of the velocity produced by the first step. Finally we evolve the system by the heat flow with Neumann boundary conditions for a small time $h$. The convergence of this method has been proved in [Benfatto and Pulvirenti 1986] in the case of the half-plane.

This class of ideas has been used for the numerical simulations presented in the sequel.

\section{Description of numerical schemes for SPH and VMs}

In the present section the numerical schemes for SPH and the VM are described. Different formulations are available in the literature; here two are briefly recalled in the context of a viscous Newtonian fluid. Further details can be found in [Graziani et al. 1995; Federico et al. 2012].

3.1. Smoothed-particle hydrodynamics formulation. In this section a SPH scheme is briefly described. The fluid is assumed to be barotropic and weakly compressible and the reference equations are the Navier-Stokes equations. The flow domain $\Omega$ is bounded by the surface $\partial \Omega_{b}$ of the solid body and by the lateral walls, where a simple free-slip condition is enforced. The lateral walls are far enough from the body to avoid significant effects on it. No-slip boundary conditions are enforced on the body surface.

As discussed in [Molteni et al. 2007; Antuono et al. 2010] different state equations, $p=f(\rho)$, can be used in the SPH scheme to model weakly compressible fluids. However, as shown below, only with the choice $\alpha=0$ it is possible to link (2-22) with the equation used in the classical SPH models. Indeed, (2-26) does not preserve the momentum conservation of the particle system, leading to a less robust and less stable numerical scheme. Therefore, here, a simple quadratic state equation (that is, $\alpha=0$ according to Section 2.1) is used to match the pressure and density field:

$$
p=A \rho^{2}+B=\frac{c_{0}^{2}}{2 \rho_{0}}\left(\rho^{2}-\rho_{0}^{2}\right)+p_{0} .
$$

Here, $c_{0}, \rho_{0}$, and $p_{0}$ are the speed of sound, the density, and the pressure with 
the fluid at rest, respectively. It is important to underline that the constant $B=$ $\left(p_{0}-c_{0}^{2} \rho_{0} / 2\right)$ is used for the purpose of numerical stability to avoid the region where the so-called tensile instability can develop [Swegle et al. 1995]. The speed of sound, $c_{0}$, is set in order to guarantee density variation smaller than $0.01 \rho_{0}$, or, in other words, the Mach number, Ma, of the simulation is less than 0.1. Indeed, as shown in [Majda 1984], in the limit for Ma going to zero the solution of the isentropic Euler equation (2-1) converges to the incompressible one (2-2).

Given a set of particles each characterized by its own mass $m_{i}$, the particle densities can be expressed through the distribution (2-23). In contrast with the method as presented in Section 2, we find it convenient to choose different masses $m_{i}$ in place of identical $m_{i}=1 / N$; therefore (2-23) becomes

$$
\rho_{i}=\sum_{j} \delta_{\epsilon}\left(x_{i}-x_{j}\right) m_{j}
$$

where $\delta_{\epsilon}=\delta_{\epsilon}\left(x_{j}-x_{i}\right)$ is the kernel function. In this work a renormalized Gaussian kernel (see, for example, [Molteni and Colagrossi 2009]) has been used. $\delta_{\epsilon}$ has a compact support of radius $3 \epsilon$, where $\epsilon$ is the smoothing length.

Deriving in time (3-2) we get the continuity equation in the SPH formalism:

$$
\dot{\rho}_{i}=\sum_{j}\left(u_{i}-u_{j}\right) \cdot \nabla_{i} \delta_{\epsilon}\left(x_{i}-x_{j}\right) m_{j} .
$$

The symbol $\nabla_{i}$ indicates differentiation with respect to the position of the $i$-th particle; for the sake of simplicity in what follows $\nabla_{i} \delta_{\epsilon}\left(x_{i}-x_{j}\right)$ will be indicated just with $\nabla_{i} \delta_{\epsilon}$. From the numerical point of view, the evaluation of the density through time integration of (3-3) is preferable with respect to the direct use of (3-2) and becomes crucial when dealing with free-surface flows (see, for example, [Colagrossi et al. 2009]).

In this section we make use of useful new relations, consequences of the energy conservation. Indeed, for an isentropic fluid the sum of the kinetic energy $\mathscr{E}_{k}$ and the internal energy $\mathscr{E}_{i}$ of the particles system is conserved:

$$
\dot{\mathscr{E}}_{k}+\dot{\mathscr{E}}_{i}=\sum_{i} m_{i} u_{i} \dot{u}_{i}+\sum_{i} m_{i} \frac{p_{i}}{\rho_{i}^{2}} \dot{\rho}_{i}=0
$$

By substituting (3-3) in (3-4) and using the kernel property

$$
\nabla_{i} \delta_{\epsilon}=-\nabla_{j} \delta_{\epsilon}
$$

the acceleration of the $i$-th particle is given by

$$
\dot{u}_{i}=-\sum_{j}\left(\frac{p_{i}}{\rho_{i}^{2}}+\frac{p_{j}}{\rho_{j}^{2}}\right) \nabla_{i} \delta_{\epsilon} m_{j} .
$$


When we use this equation for $\dot{\rho}_{i}$ and $\dot{u}_{i}$, the SPH scheme reads

$$
\left\{\begin{array}{l}
\dot{\rho}_{i}(t)=-\sum_{j}\left(u_{j}-u_{i}\right) \cdot \nabla_{i} \delta_{\epsilon} m_{j}, \\
\dot{u}_{i}(t)=-\sum_{j}\left(\frac{p_{i}}{\rho_{i}^{2}}+\frac{p_{j}}{\rho_{j}^{2}}\right) \nabla_{i} \delta_{\epsilon} m_{j}+\frac{v}{\rho_{0}} \sum_{j} \pi_{i j} \nabla_{i} \delta_{\epsilon} m_{j}, \\
\dot{x}_{i}(t)=u_{i}(t),
\end{array}\right.
$$

where $\rho_{i}, p_{i}, u_{i}$, and $m_{i}$ are, respectively, the density, pressure, velocity, and mass of the $i$-th particle, while $v$ denotes the fluid kinematic viscosity.

Thanks to the use of (3-4) the exact momentum conservation in (3-6) is guaranteed regardless of the state equation adopted. Furthermore, setting $v=0, m_{j}=1 / N$, and $p=\rho^{2} / 2$ in (3-6), then (2-22), discussed in Section 2.3, is recovered.

The viscous forces are modeled through the viscous formula of [Monaghan and Gingold 1983], which preserves both linear and angular momenta:

$$
\pi_{i j}=2(n+2) \frac{\left(u_{j}-u_{i}\right) \cdot\left(x_{j}-x_{i}\right)}{\left\|x_{j}-x_{i}\right\|^{2}},
$$

where $n$ is the spatial dimension of the problem at hand. For $\epsilon \rightarrow 0$ the system (3-6) converges to the Navier-Stokes equations (see, for example, [Colagrossi et al. 2011]).

The fluid particles are initially positioned using the algorithm described in [Colagrossi et al. 2012]. Thanks to this procedure, at the initial instant all particles have approximately the same volume, namely $V_{0}$, which is equal to the fluid domain volume divided by the number of fluid particles. Consistently, the particle mean spacing is denoted by $\Delta x=V_{0}^{1 / n}$. The average number of particles in the kernel support is set by choosing the ratio $\epsilon / \Delta x$. In the present work $\epsilon / \Delta x$ is set equal to 1.33 , which in two dimensions corresponds to about 50 interacting particles.

Along with the volume distribution, the initial pressure and the velocity fields are prescribed as well. The initial density distribution $\rho_{i}\left(t_{0}\right)$ is evaluated by means of the state equation and the particle masses are computed through the equation $m_{i}=V_{0} \rho_{i}\left(t_{0}\right)$. The mass of the $i$-th particle remains constant during the time evolution ensuring the total mass conservation of the particle system.

\subsubsection{Enforcement of the solid-boundary condition through a ghost-fluid method.} In the present work the ghost-fluid technique is used to enforce proper boundary conditions on the body surface. Specifically, the solid domain is modeled through a set of "imaginary particles" (hereinafter denoted as ghost particles and labeled with the subscript $s$ ) and all the fluid fields (that is, velocity and pressure) are extended on these fictitious particles through proper mirroring techniques. Different mirroring techniques are adopted to enforce different boundary conditions (for example, Dirichlet or Neumann conditions). To this end, the solid surface is 
discretized in equispaced body nodes and a layer of ghost particles is disposed in the solid region. The ghost particle positions have been obtained by using the technique described in [Marrone et al. 2011]. The pressure and velocity energy assigned to the fixed ghost particles, namely $\left(u_{s}, p_{s}\right)$, are computed by using the values obtained at specific interpolation nodes internal to the fluid and uniquely associated with the fixed ghost particles. Hereinafter, the interpolated values are indicated through $\left(u^{*}, p^{*}\right)$.

The pressure field $p_{s}$ is mirrored on the fixed ghost particles to enforce the Neumann condition

$$
\frac{\partial p}{\partial n}=\rho\left(f \cdot n-\dot{u}_{b} \cdot n\right)
$$

where $f$ is a generic body force and $u_{b}$ is the velocity of the solid boundary (for details see [Marrone et al. 2011]). This leads to

$$
p_{s}=p^{*}+\frac{\partial p}{\partial n} \cdot\left(x^{*}-x_{s}\right) .
$$

The velocity field is the object of a specific treatment. The ghost velocity $u_{s}$ depends on both $u^{*}$ and $u_{b}$, the latter being the velocity of the nearest body node. De Leffe et al. [2011] found that different mirroring techniques have to be used to evaluate $\langle\nabla \cdot u\rangle$ and $\left\langle\nabla^{2} u\right\rangle$ and to avoid inconsistencies and loss of accuracy. The specific mirroring techniques treat differently the components of $u^{*}$ in the normal and tangential directions to the solid surface. The velocity-divergence operator is convergent and consistent if the normal component of $u^{*}$ is mirrored in the frame of reference of the solid profile (see [Colagrossi and Landrini 2003]), leaving the tangential component unaltered. Conversely, to evaluate $\left\langle\nabla^{2} u\right\rangle$, the velocity field has to be mirrored to approximate no-slip conditions along the solid bodies. A common way is to reverse the tangential component with a linear extrapolation, leaving the normal one unaltered. The consistency of this second mirroring procedure has been discussed in [Macià et al. 2011].

Using the momentum exchange between fluid and ghost particles it is possible to evaluate the global loads acting on the solid bodies:

$$
\boldsymbol{F}_{\text {fluid-solid }}=\sum_{i \in \text { fluid }} \sum_{j \in \text { solid }}\left(-m_{i}\left(\frac{p_{j}}{\rho_{j}^{2}}+\frac{p_{i}}{\rho_{i}^{2}}\right)+v \frac{m_{i}}{\rho_{0}} \pi_{i j}\right) \nabla_{i} \delta_{\epsilon} m_{j},
$$

in which the first term on the right-hand side represents the pressure component and the second acts as the viscous component of the stress tensor.

3.2. Viscous VM with operator splitting technique. In order to analyze the vortex shedding from the body, the mathematical model can be equivalently recast in terms of the vorticity transport equation. This approach allows for removing the 
pressure from the unknown variables and for the implicit enforcement of the farfield boundary conditions.

In the case of a two-dimensional flow, the governing equation is written as

$$
\frac{D \omega}{D t}=\left(\partial_{t}+u \cdot \nabla\right) \omega(x, t)=v \nabla^{2} \omega \text { for all } x \in \Omega,
$$

where $D / D t$ denotes the material derivative.

Let us now briefly describe some detail of the numerical procedure for the solution of the governing equations. The governing equation for the vorticity field (3-10) accounts for the simultaneous advection and diffusion of this quantity. According to the well-known operator splitting approach [Chorin 1973], these two steps are separately accounted for in the computational procedure. The approximate solution of the governing equation is obtained by the sequential solution of the diffusive "Stokes" step followed by an inviscid "Euler" step for the vorticity advection. The Stokes step represents the pure diffusion of the vorticity:

$$
\frac{\partial \omega}{\partial t}=v \nabla^{2} \omega
$$

in the absence of motion combined with a vorticity generation on the solid boundary to restore the no-slip boundary condition. Indeed, the presence of a body within the flow field, requires to model the vorticity generation process on the solid walls which allows for the vortex shedding effects. Therefore, vorticity is generated on the body boundary to enforce the no-slip condition.

In the Euler step an inviscid fluid is considered where the vorticity of each particle is conserved along its Lagrangian motion:

$$
\frac{D \omega}{D t}=0 .
$$

After the splitting of the governing equation, it is clear that an appropriate procedure both for the diffusion and for the advection of the vorticity must be devised. The natural choice for the numerical solution scheme appears now as a viscous $V M$ which is based on the original papers [Chorin 1973; 1978] together with a deterministic diffusion algorithm [Benson et al. 1989] (see [Graziani et al. 1995] for more details about the adopted computational procedure).

3.2.1. Particle discretization of the vorticity field. The VM discretizes the vorticity field $\omega(x, t)$ and replaces it with a set of $N_{\text {vor }}$ point vortices with circulation $\Gamma\left(x_{j}, t\right)$ (which we write as $\Gamma_{j}(t)$ below):

$$
\omega(x, t)=\sum_{j=1}^{N_{\text {vor }}} \Gamma_{j}(t) \delta_{\epsilon}\left(x-x_{j}\right),
$$


where the spatial distribution has been regularized as already mentioned in the second section. The evolution of the vorticity field is now computed through the advection and the diffusion of the point vortices. The adoption of the vorticity as the dependent variable avoids the discretization of the irrotational regions while allowing for reserving the computational resources to the vortical zones. This is a major difference with respect to other commonly used computational schemes which require the discretization of the whole solution domain (as for the SPH method described above). At the same time, the adoption of an integral representation for the velocity field yields the exact enforcement of the far-field boundary conditions.

One of the drawbacks of the purely Lagrangian VMs stems from the excessive clustering or rarefaction of the vortex particles which requires a, somewhat nonphysical, redistribution procedure. In our case the adopted diffusion scheme avoids this possibility because a computational grid is used, only for this step, and each particle gives its diffusive contribution to the circulation of the new particles located on regular mesh nodes. At the end of the Stokes step the former set of vortex particles is replaced by that defined in the nodes of the computational mesh.

Therefore, the present hybrid Eulerian-Lagrangian computational scheme allows for the convenient evaluation of the particles' advection as well as for the efficient redistribution of the vorticity during diffusion.

We briefly recall the essential details of the four involved substeps: integral representation of the velocity field, vorticity generation, diffusion, and advection.

(1) Integral representation of the velocity field. By starting from the Helmholtz decomposition of the velocity field and considering the incompressibility constraint and the impermeability condition on $\partial \Omega$, the velocity field can be expressed by

$$
u\left(x^{\star}\right)=u_{\infty}+\int_{\partial \Omega} u_{\tau}(x) K\left(x, x^{\star}\right) d l+\int_{\Omega} \omega(x) K\left(x, x^{\star}\right) d S,
$$

where the operator $K$ has been defined in Section 2. Equation (3-14) is a way to represent the domain-dependent Green's function.

The unknown tangential velocity component $u_{\tau}$ appearing in (3-14) must be evaluated by solving an integral equation obtained by locating $x^{\star}$ on the body surface and discretizing it on $\partial \Omega$ :

$$
\begin{aligned}
& \frac{u_{i \tau}}{2}=u_{\infty \tau}+\sum_{k} u_{k \tau} \int_{\partial \Omega_{k}} \frac{\partial G}{\partial n}\left(x_{k}, x_{i}\right) d l_{k}+\tau_{i} \cdot \sum_{j} \Gamma_{j} K\left(x_{j}, x_{i}\right) d S_{j} \\
& \text { for all } x_{i} \in \partial \Omega \text {. }
\end{aligned}
$$

The last term in (3-15) is the tangential velocity on the body surface induced by the vortices. In order to evaluate it, the multipole expansion for the kernel $K$ is adopted with reduced computational cost (see [Van Dommelen and Rundensteiner 
1989]). The numerical solution of (3-15) requires a specific algorithm; details can be found in, for example, [Graziani and Landrini 1999].

At this point the enforcement of the no-slip boundary condition can be taken into account through the next step.

(2) Vorticity generation on the solid body profile. In order to satisfy the no-slip boundary condition, the adopted operator splitting approach must be complemented, in the presence of a solid body, with the vorticity generation step. In fact, the flow adherence condition is violated by the velocity field evaluated in the previous step; therefore a vortex sheet is required on the body contour whose circulation density, $\gamma$, produces the required velocity jump in the tangential velocity:

$$
\gamma\left(x^{\star}\right)=u_{b \tau}\left(x^{\star}\right)-\left(u_{\tau}\left(x^{\star}\right)+u_{\infty \tau}\right), \quad x^{\star} \in \partial \Omega,
$$

where $u_{\tau}$ is perturbation tangent velocity to the body wall evaluated through the integral equation in the first step and $u_{b \tau}$ is the tangential component of the body velocity. Here, $x^{\star}$ represents a generic point on $\partial \Omega$. This source of vorticity will be diffused within the flow field during the following step.

(3) Vorticity diffusion. The viscous effects are accounted for in the Stokes step, where the fluid velocity is neglected and the diffusion of the field vortices as well as of the wall vorticity is evaluated by solving (3-11).

The point vortex approximation can be conveniently inserted into the integral representation for the vorticity field deriving from (3-11). This equation is formally identical to the heat transfer equation and the same fundamental solution can be assumed.

The point vortex approximation (3-13) allows for a very simple and efficient evaluation of the diffusion after one time step $\Delta t_{d}=t-t_{0}$ :

$$
\omega\left(x^{\star}, t\right)=\sum_{j=1}^{N_{\text {vor }}} \frac{\Gamma_{j}\left(t_{0}\right)}{4 \pi v \Delta t_{d}} \exp \left(-\frac{\left|x_{j}-x^{\star}\right|^{2}}{4 v \Delta t_{d}}\right), \quad x^{\star} \in \text { regular meshes. }
$$

Each vortex particle, which is freely moving during the advection step and occupies the position $x_{j}$, gives its diffusive contribution to the nodes $x^{\star}$ of the properly defined grid according to the exact Lamb-Oseen solution. Therefore, after the diffusion step has been completed, the new set of particles located in the nodal positions replaces the former and an automatic and physical redistribution of the vortices occurs (the artificial viscosity due to other interpolation schemes is here absent). A piecewise constant distribution is assumed to translate vorticity into circulation in order to obtain the new vortex particles.

A few words can be spent on the diffusion of the vortex sheet on the body contour. In particular, according to the Chorin-Marsden decomposition [Chorin 1978], the vorticity derivative normal to the wall is assumed to vanish in order to satisfy the 
no-slip condition after the diffusion. Hence the vorticity of the particles close to the body is reflected back into the field in order to satisfy the Neumann condition. The application of the reflection procedure, which is an expression of the image method, is exact only for flat boundaries and gives acceptably approximated results when small curvature values are involved (otherwise the complete expression of the integral representation for the solution must be considered and solved). The vorticity reflection must be enforced only for the particles which are closest to the wall, because beyond a given distance the effect of the body is not felt, and each vortex behaves as in free space. For this reason a boundary fitted grid is assumed around the body in order to evaluate the diffusion of the closest particles while a Cartesian grid is chosen for the diffusion of the remaining ones.

(4) Advection step. The inviscid evolution of the vortical particles is described by the Euler equation (3-12) which states the material behavior of the transported quantity. Therefore, each vortex carries around its circulation without diffusion. In order to evaluate the advection step, the velocity field must be computed to obtain the new position of the vortical particles. The velocity field evaluation turns out to be the most expensive part of the whole numerical solution. In order to satisfy (3-12), each vortex is displaced by the fluid velocity according to

$$
\dot{x}_{j}(t)=u_{j}(t), \quad j=1, \ldots, N_{\mathrm{vor}},
$$

where $x_{j}(t)$ and $u_{j}(t)$ are the unknown position and velocity of each particle at time $t$, respectively. The no-flow boundary condition on the solid walls is associated with (3-18).

The velocity field $u_{j}(t)$ of the new set of vortices obtained by the diffusion step is evaluated using (3-14). In the framework of the adopted VM, the latter can be evaluated as

$$
u_{i}=u_{\infty}+\sum_{\substack{1 \leq j \leq N_{\text {vor }} \\ j \neq i}} \Gamma_{j} K_{\epsilon}\left(x_{i}, x_{j}\right), \quad x_{i} \in \Omega \cup \partial \Omega,
$$

where the $K_{\epsilon}$ is a regularized version of the kernel $K$ (see, for example, [Graziani et al. 1995]). We underline that in (3-19) the summation includes also the vortices on boundary $\partial \Omega$ needed to enforce the boundary conditions $\left(u-u_{b}\right) \cdot n=0$ and $\left(u-u_{b}\right) \cdot \tau=0$. The sum of the incoming flow $u_{\infty}$ and of the perturbation velocity yields the total velocity which allows for the vortex displacement to be evaluated. The operation count for the computation of the velocity of each particle is evidently given by $N_{\text {vor }}^{2}$ if (3-19) is directly used. In order to achieve a faster solution, the flow domain is recursively subdivided and a multipole expansion for the kernel $K$ is adopted with a computational cost of order $N_{\text {vor }} \log \left(N_{\text {vor }}\right)$, which is comparable with the performance of other efficient numerical techniques (for details see 
[Van Dommelen and Rundensteiner 1989]). This step is the most expensive part of the numerical procedure. The same approach has been used for the solution of free surface flows in [Graziani and Landrini 1999].

The numerical integration of (3-18) is performed by means of a fourth-order Runge-Kutta algorithm.

The adopted computational scheme has been shown to converge to the solution of the Navier-Stokes equations with a splitting error proportional to the time step $\Delta t$, which is therefore the relevant quantity for the overall accuracy. The numerical solution is then organized as a repeated sequence of advection, generation, and diffusion steps, each one of which can be solved in the most appropriate way. To be more specific, due to the possibly different advection and diffusion time scales, a suitable value of the time increment could be chosen for each step in order to obtain the required accuracy (for example, the time step for the diffusion could be an integral multiple of that for the advection).

3.2.2. Evaluation of the hydrodynamic loads. In order to analyze the interaction between the flow field and the body motion, the hydrodynamic force must be evaluated. A possible approach is based on the integration of the wall stress vector which can be written for a two-dimensional incompressible flow as

$$
\boldsymbol{F}=\int_{\partial \Omega}(-p n+\mu \omega \tau) d l,
$$

where $n$ is the outer normal to the body and $\tau$ is the anticlockwise tangent.

The pressure field, which is now required in order to evaluate the hydrodynamic force, must be computed as it is described below. By writing the Navier-Stokes equations on the body boundary and by taking the tangential projection, the derivative of the pressure can be expressed as

$$
\frac{1}{\rho} \frac{\partial p}{\partial s}=-\tau \cdot \dot{u}+v \frac{\partial \omega}{\partial n} .
$$

Due to the no-slip condition the fluid acceleration $\dot{u}$ on the wall must coincide with that of the body. The pressure on the body is then obtained by integrating (3-21) and the arbitrary integration constant does not affect the force. On occurrence, the hydrostatic contribution could be added either to the pressure or directly to the resultant force.

Alternatively the hydrodynamic load can be evaluated through the vorticity moments as described in [Graziani and Bassanini 2002].

As it is usual with VMs (see, for example, [Kinney and Cielak 1977]), the last term in (3-21), that is, that related to the vorticity flux from the body, is expressed in terms of the circulation density $\gamma$ generated on the wall during the actual step 
according to

$$
v \frac{\partial \omega}{\partial n}(s)=-\frac{\gamma(s)}{\Delta t} .
$$

To resume, the pressure on the wall is obtained by integrating (3-21), then the force is yielded by an integration according to (3-20).

\section{Numerical results: flow past a fixed circular cylinder}

The classical evolution of the flow past a circular cylinder is here considered. Two Reynolds numbers $\operatorname{Re}=140$ and $\operatorname{Re}=1000$ are simulated both with SPH as well as with the VM described in the previous sections.

Conversely to the VM, for the SPH model the definition of a proper algorithm for the inflow and outflow particles is required (see, for example, [Federico et al. 2012]). Using such algorithm the new inflow particles which enter in the fluid domain are added at the end of the particles' array.

For the VM, since the fluid is treated as incompressible, the flow can be started impulsively without any numerical problems. This is not possible with SPH due to the weakly compressible model adopted. In this case the cylinder is smoothly accelerated with a prescribed time law. The problem is further complicated for SPH by the treatment of the acoustic shock waves, generated during the acceleration stage. The pressure fields at the inflow/outflow boundaries are corrected so as to let the acoustic wave cross them as described in [Lastiwka et al. 2009]. At $\operatorname{Re}=140$ it is well known that the wake is not symmetric and alternate sign vorticity is released, producing the characteristic Kármán vortex sheet. The top portion of Figure 1 shows the streakline obtained experimentally using an electrolytic precipitation of a colloidal smoke [van Dyke 1982]. Thanks to the pure Lagrangian nature of SPH it is very easy to extract the streakline from the wake. Indeed it is sufficient to plot only the upper part of the particles' array which represents the particles that remain for a longer time in the fluid domain before leaving it through the outflow boundary (see the bottom portion of Figure 1).

The SPH simulation has been performed with a spatial resolution of $D / \Delta x=$ 140 , with $D$ being the cylinder diameter and $\Delta x$ the particles' size. The total number of particles required is 4.4 million. In Figure 1 only the first 700,000 particles stored in the array have been plotted with black dots. This kind of visualization is not trivial to obtain with the VM described in the previous section since the diffusion process on the regular mesh destroys the Lagrangian particle path. On the other hand, we show later that the VM allows a prediction of the vorticity field with a larger accuracy with respect to the SPH method. It is important to underline that a small perturbation on the vertical motion of the cylinder has been forced in 

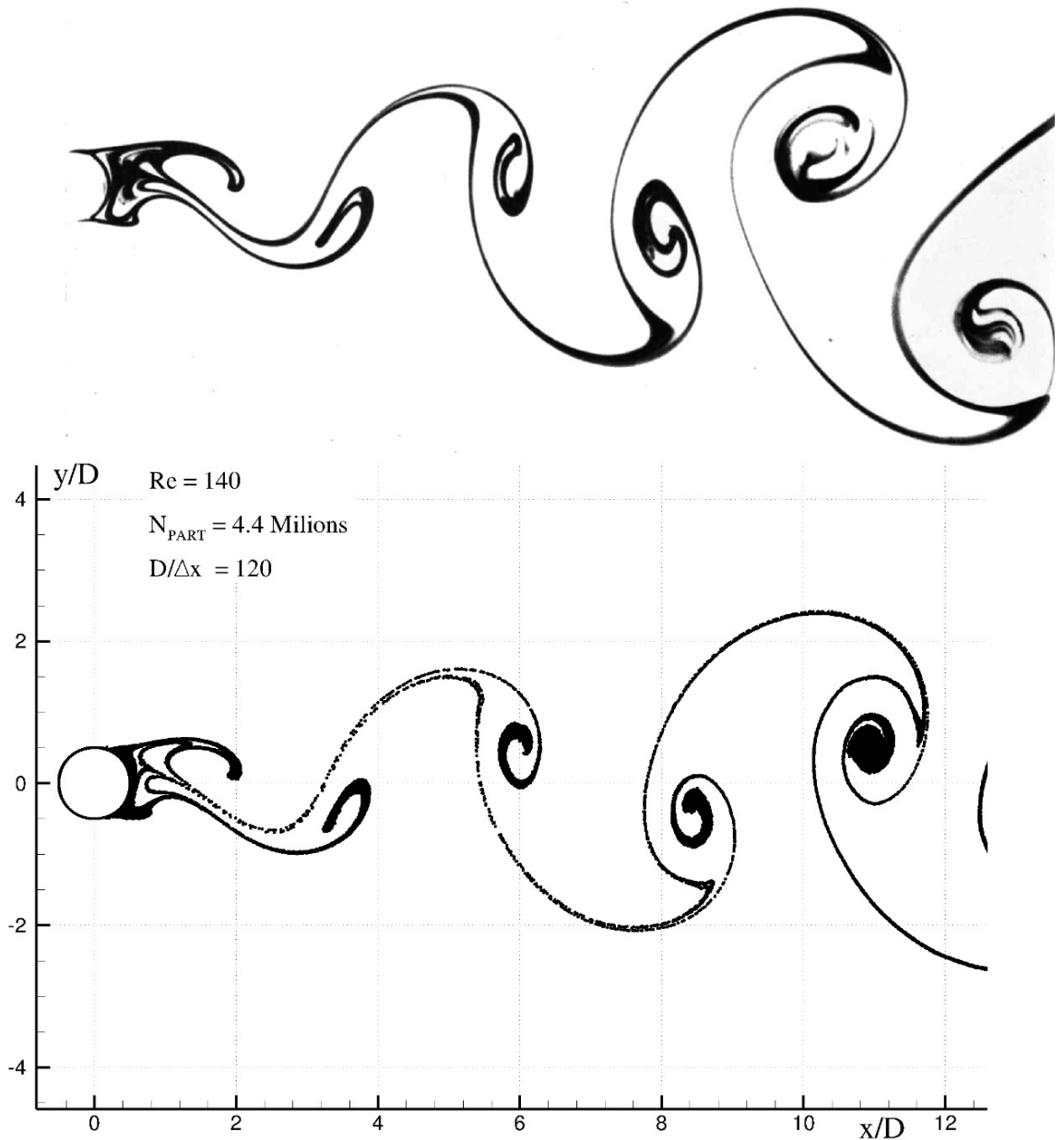

Figure 1. Streakline at $\mathrm{Re}=140$. Top: experimental photograph by Sadatoshi Taneda [van Dyke 1982]. Bottom: SPH simulation.

the vortex model to break the symmetry of the numerical solution and to yield the vortex shedding. This operation is not necessary with the SPH solver for which the symmetric solution is automatically lost after a time which depends on the spatial resolution adopted. The more particles are used, the more time is needed to start the vortex shedding.

Figure 2 shows a comparison between SPH and VM on the drag and lift coefficients. The two different solvers are in fairly good agreement. For the VM the cylinder surface is discretized with 300 panels, therefore the spatial resolution is similar to the SPH one. At the end of the simulation 180,000 vortices are present in the fluid domain. The VM simulation takes one hour on an eight-core CPU (Xeon 

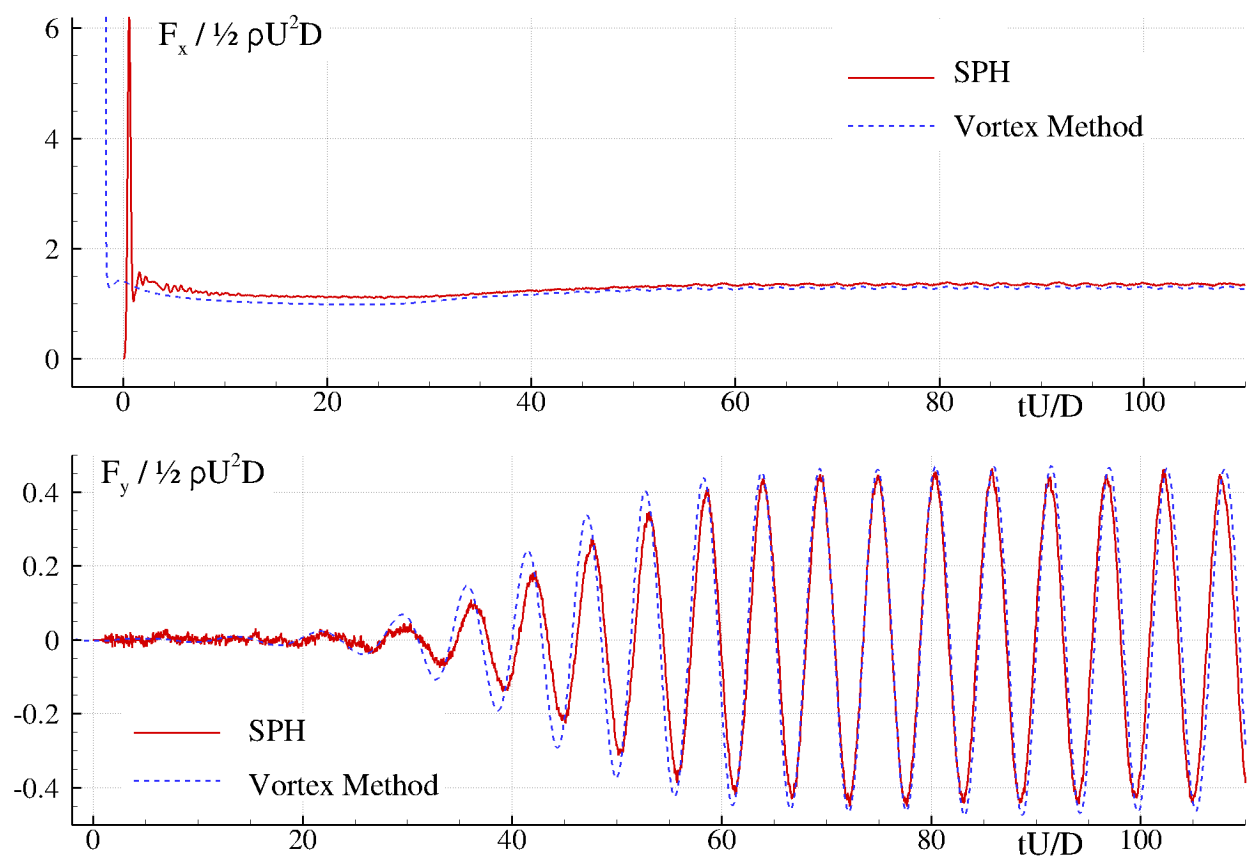

Figure 2. Comparison of the drag and lift forces as predicted by SPH (solid line) and VM (dashed line) for a circular cylinder at $\operatorname{Re}=140$.

E5410 2.33GHz), compared with the four days required for SPH. For this kind of flow SPH has very high CPU costs. However, it must be underlined that the SPH model can easily simulate complex two and three-dimensional violent free-surface flows (see, for example, [Marrone et al. 2011]), which is not the case for the VM.

Finally the case of $\operatorname{Re}=1000$ is considered. Incidentally, we note that twodimensional simulations at this Re value represent a mere numerical test since in reality the flow is fully three-dimensional. Figure 3 shows the vorticity field evaluated by SPH and VM. The SPH vorticity field is quite noisy; besides this the vorticity field of the two solvers are very similar in terms of intensity and shape of the vortical structures.

To solve the flow at this Reynolds number, the spatial resolution has been increased to $D / \Delta x=200$ for SPH and similarly in the VM the cylinder surface has been discretized with 600 panels. The total number of particles adopted for SPH is five million and the simulation takes 26 hours solving a wake 12 diameters long. For the VM the number of vortices at the end of the simulation is 3.3 million, and the simulation takes five days solving a wake 52 diameters long, much longer than the SPH one. The whole wake calculated by the VM is reported in Figure 4 for two different time instants. 

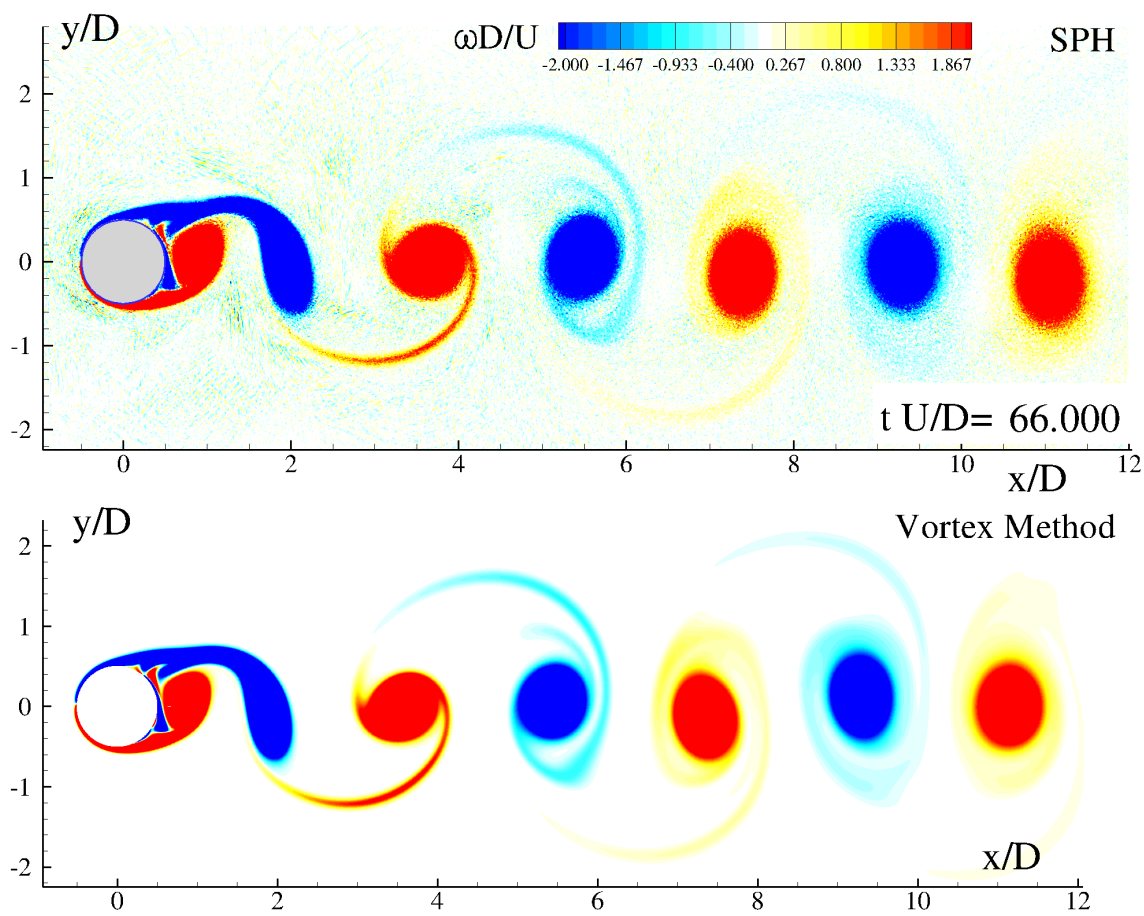

Figure 3. Vorticity field at $\operatorname{Re}=1000$ (two-dimensional simulation) as predicted by SPH (top) and VM (bottom).
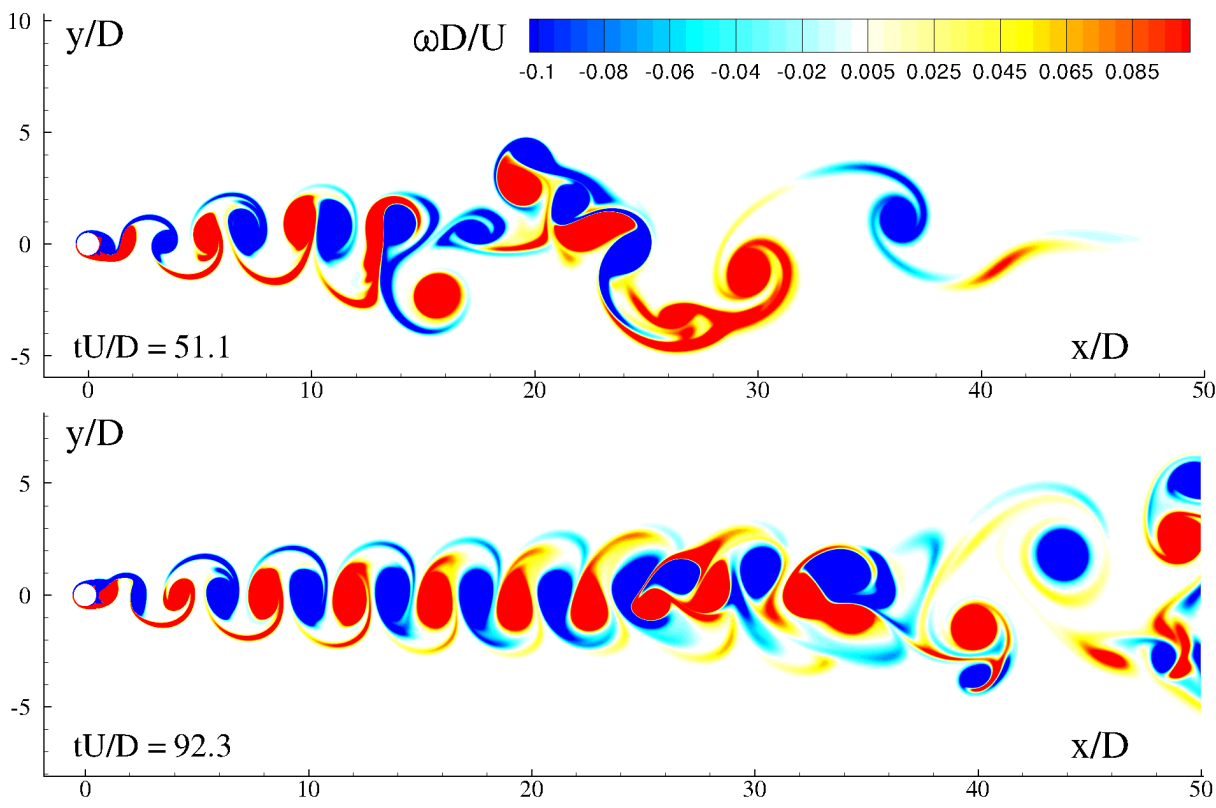

Figure 4. Wake field at $\mathrm{Re}=1000$ (two-dimensional simulation) evaluated by the $\mathrm{VM}$ at times $t U / D=51.1$ and $t U / D=92.3$. 

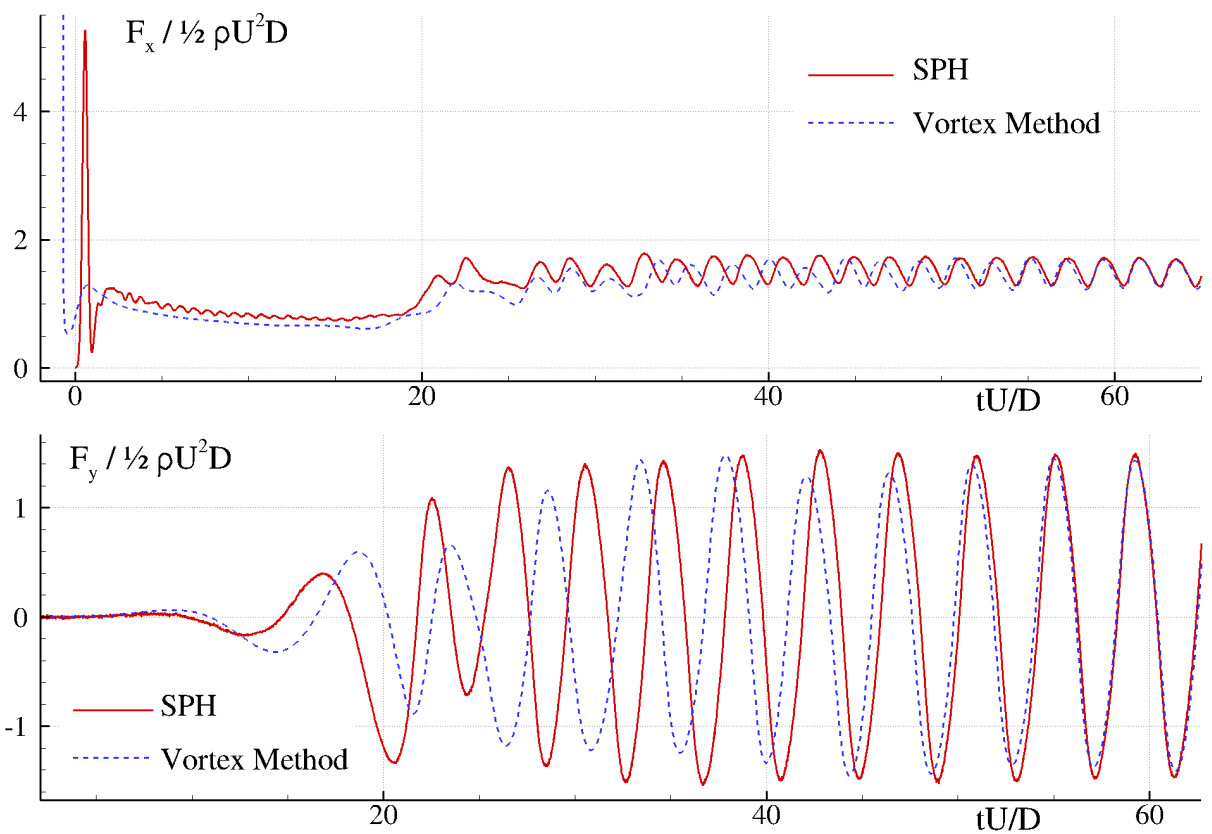

Figure 5. Comparison of the drag and lift forces as predicted by SPH (solid line) and VM (dashed line) for a circular cylinder at $\operatorname{Re}=1000$ (two-dimensional simulation).

The use of different fluid domain lengths adopted for the two solvers reflects on the global loads acting on the cylinder depicted in Figure 5. However, the comparison on the loads remains quite fair when the periodic regime has been reached.

\section{Conclusions}

In this work the use of particle methods for solving fluid dynamic problems is discussed. Models are analyzed theoretically in the context of weakly compressible and incompressible inviscid fluid dynamics. The convergence of the regularized solutions is discussed by using Vlasov dynamics. Numerical formulations for both smoothed-particle hydrodynamics and the vortex model are described for solving viscous weakly compressible and incompressible flows. Application to the flow around a circular cylinder is discussed for both methods considering Reynolds numbers 140 and 1000. The numerical results obtained with the two solvers are compared and the benefits and drawbacks of the different formulations are highlighted. 


\section{Acknowledgements}

The research leading to these results received funding from the Flagship Project RITMARE (Italian Maritime Research), coordinated by the Italian National Research Council and funded by the Italian Ministry of Education, Universities and Research within the National Research Program 2011-2013.

\section{References}

[Antonuccio-Delogu et al. 1996] V. Antonuccio-Delogu, U. Becciani, and F. Magugliani, "Parallelization strategies for tree N-body codes", pp. 24-32 in Applied parallel computing: Computations in physics, chemistry and engineering science, edited by J. Dongarra et al., Lecture Notes in Computer Science 1041, 1996.

[Antuono et al. 2010] M. Antuono, A. Colagrossi, S. Marrone, and D. Molteni, "Free-surface flows solved by means of SPH schemes with numerical diffusive terms", Comput. Phys. Comm. 181:3 (2010), 532-549.

[Barnes and Hut 1986] J. E. Barnes and P. Hut, "A hierarchical $O(N \log N)$ force calculation algorithm”, Nature 324:4 (1986), 446-449.

[Ben Moussa 2006] B. Ben Moussa, "On the convergence of SPH method for scalar conservation laws with boundary conditions", Methods Appl. Anal. 13:1 (2006), 29-61.

[Ben Moussa and Vila 2000] B. Ben Moussa and J. P. Vila, "Convergence of SPH method for scalar nonlinear conservation laws”, SIAM J. Numer. Anal. 37:3 (2000), 863-887.

[Benfatto and Pulvirenti 1984] G. Benfatto and M. Pulvirenti, "Generation of vorticity near the boundary in planar Navier-Stokes flows", Comm. Math. Phys. 96:1 (1984), 59-95.

[Benfatto and Pulvirenti 1986] G. Benfatto and M. Pulvirenti, "Convergence of Chorin-Marsden product formula in the half-plane", Comm. Math. Phys. 106:3 (1986), 427-458.

[Benson et al. 1989] M. G. Benson, P. G. Bellamy-Knights, J. H. Gerrard, and I. Gladwell, "A viscous splitting algorithm applied to low Reynolds number flows round a circular cylinder", Journ. Fluids and Structures 3 (1989), 439-479.

[Boldrighini and Buttà 2011] C. Boldrighini and P. Buttà, "Navier-Stokes equations on a flat cylinder with vorticity production on the boundary", Nonlinearity 24:9 (2011), 2639-2662.

[Braun and Hepp 1977] W. Braun and K. Hepp, "The Vlasov dynamics and its fluctuations in the $1 / N$ limit of interacting classical particles", Comm. Math. Phys. 56:2 (1977), 101-113.

[Chorin 1973] A. J. Chorin, "Numerical study of slightly viscous flow", J. Fluid Mech. 57:4 (1973), 785-796.

[Chorin 1978] A. J. Chorin, "Vortex sheet approximation of boundary layers", J. Comput. Phys. 27 (1978), 428-442.

[Colagrossi and Landrini 2003] A. Colagrossi and M. Landrini, "Numerical simulation of interfacial flows by Smoothed Particle Hydrodynamics", Journal of Computational Physics 191 (2003), 448475 .

[Colagrossi et al. 2009] A. Colagrossi, M. Antuono, and D. Le Touzé, "Theoretical considerations on the free-surface role in the Smoothed Particle Hydrodynamics model”, Physical Review E 79:5 (2009), 056701: 1-13. 
[Colagrossi et al. 2011] A. Colagrossi, M. Antuono, A. Souto-Iglesias, and D. Le Touzé, "Theoretical analysis and numerical verification of the consistency of viscous smoothed-particle-hydrodynamics formulations in simulating free-surface flows", Physical Review E 84 (2011), article id 026705 .

[Colagrossi et al. 2012] A. Colagrossi, B. Bouscasse, M. Antuono, and S. Marrone, "Particle packing algorithm for SPH schemes”, Comput. Phys. Commun. 183:8 (2012), 1641-1653.

[De Leffe et al. 2011] M. De Leffe, D. Le Touzé, and B. Alessandrini, "A modified no-slip condition in weakly-compressible SPH", pp. 291-297 in Proc. 6th International SPHERIC Workshop (Hamburg, 2011), edited by T. Rung and C. Ulrich, Schriftenreihe Schiffbau 658, Hamburg TUHH, 2011.

[Di Lisio 1995] R. Di Lisio, "A particle method for a self-gravitating fluid: A convergence result", Math. Methods Appl. Sci. 18:13 (1995), 1083-1094.

[Di Lisio et al. 1997] R. Di Lisio, E. Grenier, and M. Pulvirenti, "On the regularization of the pressure field in compressible Euler equations”, Ann. Scuola Norm. Sup. Pisa Cl. Sci. (4) 24:2 (1997), 227-238.

[Di Lisio et al. 1998] R. Di Lisio, E. Grenier, and M. Pulvirenti, "The convergence of the SPH method", Comput. Math. Appl. 35:1-2 (1998), 95-102.

[Dobrušin 1979] R. L. Dobrušin, "Vlasov equations", Funktsional. Anal. i Prilozhen. 13:2 (1979), 48-58. In Russian; translated in Funct. Anal. Appl. 13 (1979), 115-123.

[van Dyke 1982] M. van Dyke, An album of fluid motion, Parabolic Press, Stanford, CA, 1982.

[Federico et al. 2012] I. Federico, S. Marrone, A. Colagrossi, F. Aristodemo, and M. Antuono, "Simulating 2D open-channel flows through an SPH model", Eur. J. Mech. B Fluids 34 (2012), $35-46$.

[Graziani and Bassanini 2002] G. Graziani and P. Bassanini, "Unsteady viscous flows about bodies: Vorticity release and forces", Meccanica 37:3 (2002), 283-303.

[Graziani and Landrini 1999] G. Graziani and M. Landrini, "Application of multipoles expansion technique to two-dimensional nonlinear free-surface flows”, J. Ship Res. 43 (1999), 1-12.

[Graziani et al. 1995] G. Graziani, M. Ranucci, and R. Piva, "From a boundary integral formulation to a vortex method for viscous flows", Comput. Mech. 15 (1995), 301-314.

[Kinney and Cielak 1977] R. B. Kinney and Z. M. Cielak, "Analysis of unsteady viscous flow past an airfoil, I: Theoretical development”, AIAA Journal 15 (1977), 1712-1717.

[Koumoutsakos 2005] P. Koumoutsakos, "Multiscale flow simulations using particles", Annu. Rev. Fluid Mech. 37 (2005), 457-487.

[Lastiwka et al. 2009] M. Lastiwka, M. Basa, and N. J. Quinlan, "Permeable and non-reflecting boundary conditions in SPH”, Internat. J. Numer. Methods Fluids 61:7 (2009), 709-724.

[Lucy 1977] L. Lucy, "A numerical approach to testing the fission hypothesis", Astron. J. 82 (1977), 1013.

[Macià et al. 2011] F. Macià, M. Antuono, L. M. Gonzales, and A. Colagrossi, "Theoretical analysis of the no-slip boundary condition enforcement in SPH methods", Prog. Theor. Phys. 125:6 (2011), $1091-1121$.

[Majda 1984] A. Majda, Compressible fluid flow and systems of conservation laws in several space variables, Applied Mathematical Sciences 53, Springer, New York, 1984.

[Marchioro and Pulvirenti 1982] C. Marchioro and M. Pulvirenti, "Hydrodynamics in two dimensions and vortex theory", Comm. Math. Phys. 84:4 (1982), 483-503. 
[Marchioro and Pulvirenti 1984] C. Marchioro and M. Pulvirenti, Vortex methods in two-dimensional fluid dynamics, Lecture Notes in Physics 203, Springer, Berlin, 1984.

[Marchioro and Pulvirenti 1994] C. Marchioro and M. Pulvirenti, Mathematical theory of incompressible nonviscous fluids, Applied Mathematical Sciences 96, Springer, New York, 1994.

[Marrone et al. 2011] S. Marrone, M. Antuono, A. Colagrossi, G. Colicchio, D. Le Touzé, and G. Graziani, " $\delta$-SPH model for simulating violent impact flows", Comput. Methods Appl. Mech. Engrg. 200:13-16 (2011), 1526-1542.

[Marrone et al. 2012] S. Marrone, B. Bouscasse, A. Colagrossi, and M. Antuono, "Study of ship wave breaking patterns using 3D parallel SPH simulations", Comput. Fluids 69 (2012), 54-66.

[Mas-Gallic and Raviart 1987] S. Mas-Gallic and P.-A. Raviart, "A particle method for first-order symmetric systems", Numer. Math. 51:3 (1987), 323-352.

[Molteni and Colagrossi 2009] D. Molteni and A. Colagrossi, "A simple procedure to improve the pressure evaluation in hydrodynamic context using the SPH", Comput. Phys. Comm. 180:6 (2009), 861-872.

[Molteni et al. 2007] D. Molteni, A. Colagrossi, and G. Colicchio, "On the use of an alternative water state equation in SPH”, pp. 23-26 in 2nd SPHERIC International Workshop (Madrid, 2007), edited by A. J. C. Crespo et al., Universidade de Vigo, 2007.

[Monaghan 1992] J. J. Monaghan, "Smoothed particle hydrodynamics", Ann. Rev. of Astron. and Astroph. 30 (1992), 543.

[Monaghan 1994] J. J. Monaghan, "Simulating free surface flows with SPH", J. Comp. Phys 110:2 (1994), 399-406.

[Monaghan and Gingold 1983] J. J. Monaghan and R. A. Gingold, "Shock simulation by the particle method SPH", Journal of Computational Physics 52 (1983), 374-389.

[Neunzert 1984] H. Neunzert, "An introduction to the nonlinear Boltzmann-Vlasov equation", pp. 60-110 in Kinetic theories and the Boltzmann equation (Montecatini, 1981), edited by C. Cercignani, Lecture Notes in Math. 1048, Springer, Berlin, 1984.

[Newton 2001] P. K. Newton, The N-vortex problem: Analytical techniques, Applied Mathematical Sciences 145, Springer, New York, 2001.

[Oelschläger 1991] K. Oelschläger, "On the connection between Hamiltonian many-particle systems and the hydrodynamical equations”, Arch. Rational Mech. Anal. 115:4 (1991), 297-310.

[Swegle et al. 1995] J. W. Swegle, D. L. Hicks, and S. W. Attaway, "Smoothed particle hydrodynamics stability analysis", J. Comput. Phys. 116:1 (1995), 123-134.

[Van Dommelen and Rundensteiner 1989] L. Van Dommelen and E. A. Rundensteiner, "Fast, adaptive summation of point forces in the two dimensional Poisson equation", J. Comput. Phys 83 (1989), 126-147.

Received 5 Nov 2012. Revised 20 Feb 2013. Accepted 4 Apr 2013.

ANDREA COLAGROSSI: andrea.colagrossi@cnr.it

CNR-INSEAN, Via di Vallerano 139, I-00128 Rome, Italy

GIORGIO GRAZIANI: g.graziani@uniroma1.it

Dipartimento di Ingegneria Meccanica e Aerospaziale, Universitá di Roma "La Sapienza",

Via Eudossiana 18, I-00184 Rome, Italy

MARIO PUlVIRENTI: pulviren@mat.uniroma1.it

Dipartimento di Matematica, Universitá di Roma "La Sapienza", Piazzale Aldo Moro 2, I-00185 Rome, Italy 


\section{EDITORIAL BOARD}

ANTONIO CARCATERRA ERIC A. CARLEN

FRANCESCO DELL'ISOLA

RAFFAELE ESPOSITO

ALBERT FANNJIANG

Gilles A. FrancFort

PiERANGElo MARCATI

JEAN-JACQUES MARIGO

PETER A. MARKOWICH MARTIN OSTOJA-STARZEWSKI

PIERRE SEPPECHER

DAVID J. STEIGMANN

PAUl STEINMANN

PierRe M. Suquet

\section{MANAGING EDITORS}

MICOL AMAR

CORRADO LATTANZIO

ANGELA MADEO

MARTIN OSTOJA-STARZEWSKI

\section{ADVISORY BOARD}

ADNAN AKAY

Holm AltenbaCH

MICOL AMAR

HARM ASKES

TEODOR ATANACKOVIĆ

VICTOR BERDICHEVSKY

GuY BouchitTÉ

ANDREA BRAIDES

ROBERTO CAMASSA

MAURO CARFORE

ERIC DARVE

FELIX DARVE

ANNA DE MASI

Gianpietro Del Piero

EMMANUELE Di BENEDETTO

BERNOLD FIEDLER

IRENE M. GAMBA

SERGEY GAVRILYUK

TIMOTHY J. HEALEY

DOMINIQUE JEULIN

ROGER E. KHAYAT

CORRADO LATTANZIO

ROBERT P. LIPTON

ANGELO LUONGO

ANGEla MadeO

JUAN J. MANFREDI

CARLO MARCHIORO

GÉrard A. MAUGin

ROBERTO NATALINI

PATRIZIO NEFF

ANDREY PIATNITSKI

ERrico Presutti

MARIO PUlVIRENTI

LuCiO RuSSO

Miguel A. F. SANJUAN

Patrick SElvadurai

ALEXANDER P. SEYRANIAN

MIROSLAV ŠILHAVÝ

GUIDO SWEERS

ANTOINETTE TORDESILLAS

LEV TRUSKINOVSKY

JUAN J. L. VELÁZQUEZ

VINCENZO VESPRI

ANGELO VULPIANI msp.org/memocs

Università di Roma “La Sapienza”, Italia

Rutgers University, USA

(CO-CHAIR) Università di Roma "La Sapienza", Italia

(TREASURER) Università dell'Aquila, Italia

University of California at Davis, USA

(CO-CHAIR) Université Paris-Nord, France

Università dell' Aquila, Italy

École Polytechnique, France

DAMTP Cambridge, UK, and University of Vienna, Austria

(CHAIR MANAGING EDITOR) Univ. of Illinois at Urbana-Champaign, USA

Université du Sud Toulon-Var, France

University of California at Berkeley, USA

Universität Erlangen-Nürnberg, Germany

LMA CNRS Marseille, France

Università di Roma “La Sapienza”, Italia

Università dell' Aquila, Italy

Université de Lyon-INSA (Institut National des Sciences Appliquées), France (CHAIR MANAGING EDITOR) Univ. of Illinois at Urbana-Champaign, USA

Carnegie Mellon University, USA, and Bilkent University, Turkey

Otto-von-Guericke-Universität Magdeburg, Germany

Università di Roma "La Sapienza”, Italia

University of Sheffield, UK

University of Novi Sad, Serbia

Wayne State University, USA

Université du Sud Toulon-Var, France

Università di Roma Tor Vergata, Italia

University of North Carolina at Chapel Hill, USA

Università di Pavia, Italia

Stanford University, USA

Institut Polytechnique de Grenoble, France

Università dell'Aquila, Italia

Università di Ferrara and International Research Center MEMOCS, Italia

Vanderbilt University, USA

Freie Universität Berlin, Germany

University of Texas at Austin, USA

Université Aix-Marseille, France

Cornell University, USA

École des Mines, France

University of Western Ontario, Canada

Università dell' Aquila, Italy

Louisiana State University, USA

Università dell' Aquila, Italia

Université de Lyon-INSA (Institut National des Sciences Appliquées), France

University of Pittsburgh, USA

Università di Roma "La Sapienza", Italia

Université Paris VI, France

Istituto per le Applicazioni del Calcolo "M. Picone", Italy

Universität Duisburg-Essen, Germany

Narvik University College, Norway, Russia

Università di Roma Tor Vergata, Italy

Università di Roma "La Sapienza", Italia

Università di Roma “Tor Vergata", Italia

Universidad Rey Juan Carlos, Madrid, Spain

McGill University, Canada

Moscow State Lomonosov University, Russia

Academy of Sciences of the Czech Republic

Universität zu Köln, Germany

University of Melbourne, Australia

École Polytechnique, France

Bonn University, Germany

Università di Firenze, Italia

Università di Roma La Sapienza, Italia

MEMOCS (ISSN 2325-3444 electronic, 2326-7186 printed) is a journal of the International Research Center for the Mathematics and Mechanics of Complex Systems at the Università dell'Aquila, Italy.

Cover image: "Tangle" by @ John Horigan; produced using the Context Free program (contextfreeart.org).

\section{PUBLISHED BY}

mathematical sciences publishers

nonprofit scientific publishing

http://msp.org/

(C) 2014 Mathematical Sciences Publishers 
Mathematics and Mechanics of Complex Systems vol. 2 no. 1

Delaminated thin elastic inclusions inside elastic bodies

Alexander M. Khludnev and Günter R. Leugering

Interfaces endowed with nonconstant surface energies

23 revisited with the d'Alembert-Lagrange principle

Henri Gouin

Particles for fluids: SPH versus vortex methods

Andrea Colagrossi, Giorgio Graziani and Mario

Pulvirenti

Nonclassical continua, pseudobalance, and the law of action 71 and reaction

Gianpietro Del Piero

MEMOCS is a journal of the International Research Center for the Mathematics and Mechanics of Complex Systems at the Università dell' Aquila, Italy.

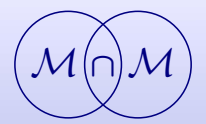

